\title{
Non-pyothorax-associated primary pleural lymphoma without pleural effusion in an immunocompetent patient: a case report and literature review
}

\author{
Chuan Shao ${ }^{1,2}$, Yongwei Guo ${ }^{3}$, Xianfa Xu ${ }^{4}$, Deng Pan $^{4}$, Bijiong Wang ${ }^{1,2}$, Yaodong Tang ${ }^{1,2}$, Shuguang Xu ${ }^{1,2}$ \\ ${ }^{1}$ Department of Respiratory Medicine, Ningbo Medical Center Lihuili Eastern Hospital, Ningbo 315040, China; ${ }^{2}$ Department of Respiratory \\ Medicine, Taipei Medical University Ningbo Medical Center, Ningbo 315040, China; ${ }^{3}$ Department of Ophthalmology, University of Cologne, \\ 50937 Cologne, Germany; ${ }^{4}$ Department of Cytopathology, Ningbo Diagnostic Pathology Center, Ningbo 315021, China \\ Correspondence to: Shuguang Xu. Department of Respiratory Medicine, Ningbo Medical Center Lihuili Eastern Hospital, Taipei Medical University \\ Ningbo Medical Center, No. 1111 Jiangnan Road, Ningbo 315040, China. Email: xushuguang2008@126.com.
}

Submitted Dec 29, 2017. Accepted for publication Apr 19, 2018.

doi: $10.21037 /$ jtd.2018.04.163

View this article at: http://dx.doi.org/10.21037/jtd.2018.04.163

\section{Introduction}

Although the pleura can be frequently invaded by lymphoma, primary pleural lymphoma is rare and relevant published literature are all case reports (1). It is usually divided into two types: primary effusion lymphoma (PEL) and pyothorax-associated lymphoma (PAL) (2). Primary pleural lymphoma without pleural effusion and history of chronic pyothorax is extremely rare and evidence-based decisions are few. Diffuse large B cell lymphoma (DLBCL) is the most common type of non-Hodgkin's lymphoma (3). Here we present a case of non-pyothorax-associated primary pleural DLBCL with pleural thickening as the only radiological manifestation. Its diagnostic procedure and therapeutic strategy were described. The possibility of lymphoma should also be considered in patients with pleural thickening, in addition to chronic inflammation and pleural mesothelioma.

\section{Case presentation}

A 65-year-old man attended our respiratory clinic with a 4-month history of mild dull pain on the right side of the chest and back. The pain was not related to body position or respiration. He denied fever, cough, weight loss or any history of chest trauma. He had a 9-year history of chronic obstructive pulmonary disease and was treated with budesonide/formoterol turbuhaler regularly. Physical examination was unremarkable. Apart from a slight decrease of white blood cell count to $2.9 \times 10^{9} / \mathrm{L}$, no other obvious abnormalities were found in his other laboratory results including erythrocyte sedimentation rate and C-reactive protein.

He was then admitted to our ward for further management. Urine and stool tests, biochemical tests, coagulation test as well as tumor biomarkers were within normal limits. Infections including tuberculosis and HIV were ruled out. Abdominal ultrasonography demonstrated no abnormalities. Contrast-enhanced computed tomography (CT) scan of the chest showed inhomogeneous pleural thickening on the right without mediastinal lymph node enlargement. The lesion showed uniform enhancement and a vessel was visible going through it (Figure 1). Pleural mesothelioma was suspected, hence CT-guided percutaneous core needle biopsy of the pleura was performed. Pathological and immunohistochemical examinations of the specimen revealed a DLBCL (Figure 2). The patient underwent bone marrow biopsy and no abnormalities were found. PET CT scan was suggested while the patient refused it for economic reasons. Following a discussion with hematologists, he was treated with chemoimmunotherapy of rituximab together with cyclophosphamide, vincristine, doxorubicin, and prednisone (R-CHOP). The patient experienced leukopenia after therapy and then recombinant human granulocyte colony stimulating factor was used. The lesion reduced after eight cycles of chemotherapy (Figure 1) and he maintained good performance status. 

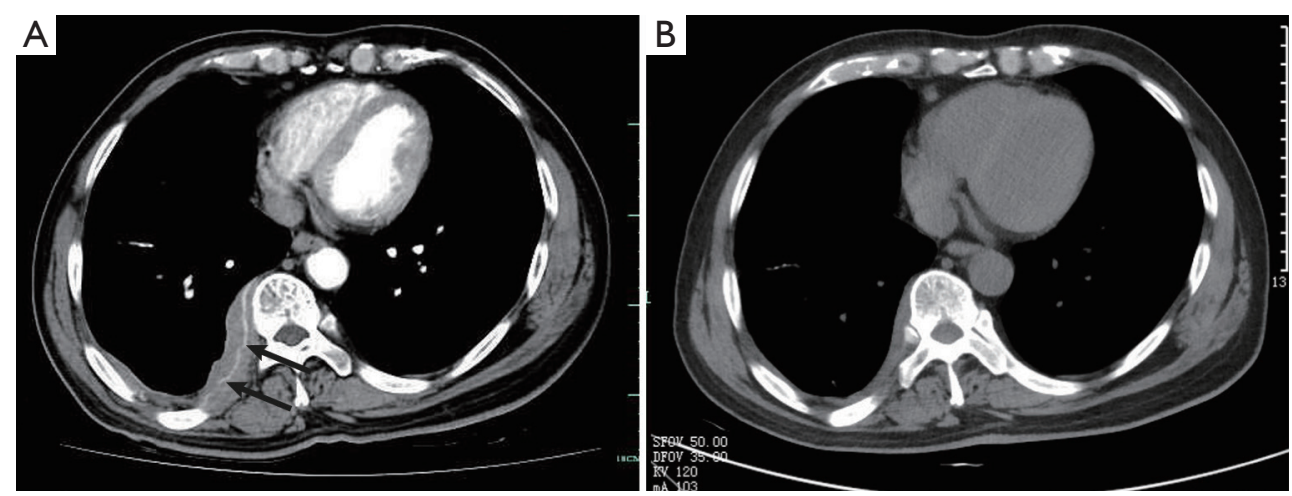

Figure 1 Images of contrast-enhanced CT scan and CT scan after chemotherapy. (A) Contrast-enhanced CT scan showed pleural thickening on the right. A vessel was enveloped by the lesion of primary pleural lymphoma as noted by arrows, which can be called "sandwich sign"; (B) CT scan after chemotherapy showed the lesion was reduced.
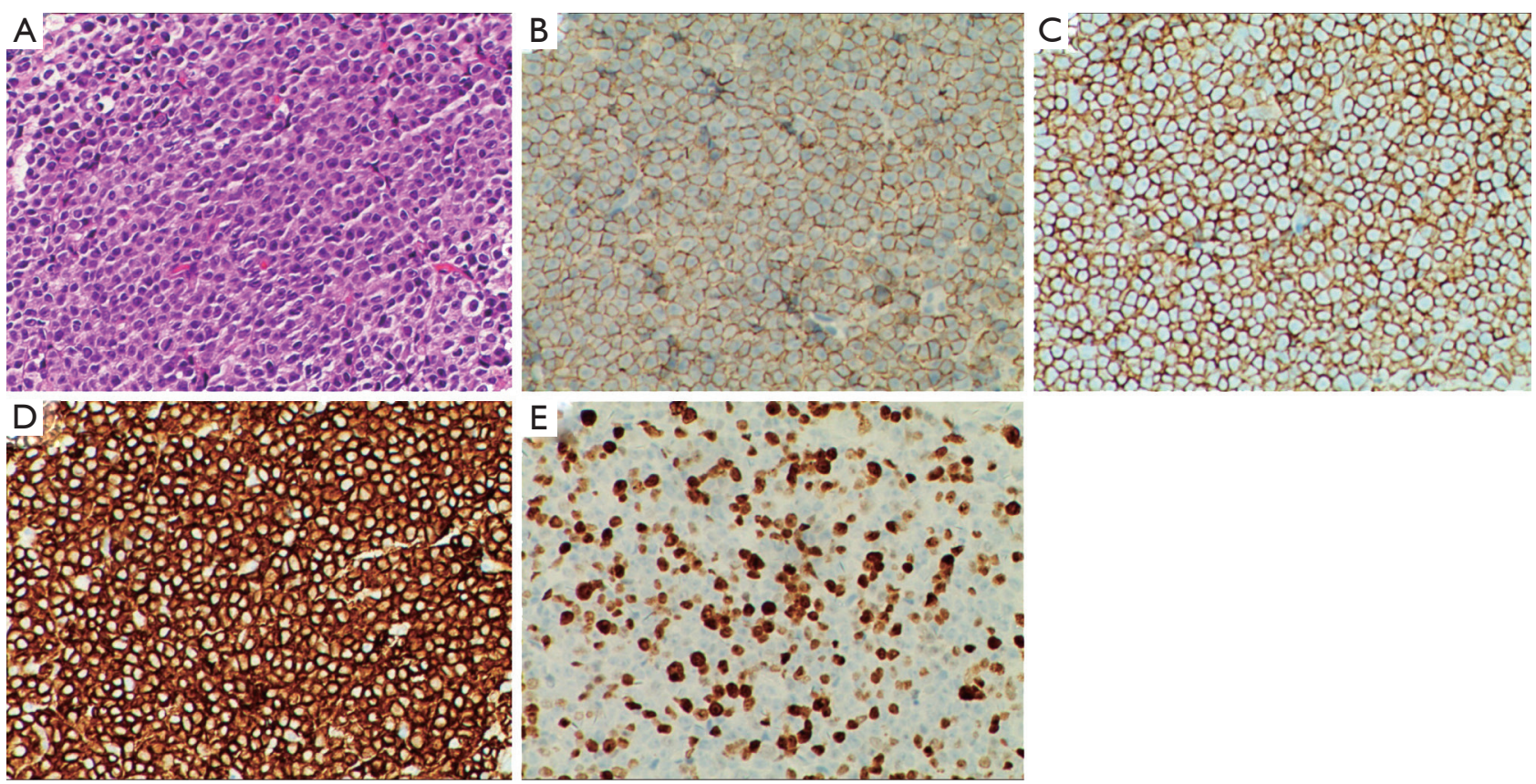

Figure 2 Histopathological and immunohistochemical findings of the primary pleural DLBCL. (A) Pathological findings of the pleural tumor showed DLBCL (H\&E staining, $\times 200)$; (B,C,D,E) immunohistochemically, the tumor cells showed immunoreactivity for CD20 (B, $\times 200)$, CD45 (C, ×200), CD79a (D, ×200) and Ki-67 (E, ×200).

\section{Discussion}

In this patient, there was no palpable lymphadenopathy although deep lymph nodes cannot be touched by physical exam. Hepato-or splenomegaly and obvious abdominal lymphadenopathy were excluded through abdominal ultrasonography. The results of bone marrow biopsy were normal and bone marrow infiltration was excluded. We had ever suggested PET-CT scan for systemic evaluation, unfortunately the patient refused it for economic reasons. As lymph nodes, liver, spleen and bone marrow are the most frequent sites lymphoma may involve, we had excluded other possible lesions with available exams except PET-CT. Therefore, based on the available results we considered it a case of primary pleural lymphoma. 
Table 1 Summary of non-pyothorax-associated primary pleural DLBCL without pleural effusion

\begin{tabular}{lccccc}
\hline Case No. & Age & Gender & Radiographic findings & Treatment & Survival time \\
\hline 1 & 76 & $\mathrm{M}$ & Mass & CT + RT & Death (5 months) \\
2 & 82 & F & Mass, bone invasion & CT $\rightarrow$ OP $\rightarrow$ CT $\rightarrow$ RT & Alive (15 months) \\
3 & 84 & F & Mass, bone invasion & CT & Death (4 months) \\
4 & 67 & F & Mass, bone invasion & CT & Alive (36 months) \\
5 & 39 & F & MT + CT & Alive (60 months) \\
6 & 17 & M & Mass, bone invasion & OP + CT & Alive (47 months) \\
7 & 48 & F & Mass, bone invasion & OP + CT + RT & Alive (8 months) \\
\hline
\end{tabular}

DLBCL, diffuse large B cell lymphoma; CT, chemotherapy; RT, radiotherapy; OP, operation.

Primary pleural lymphoma is rare, although pleural involvement is a common manifestation of secondary lymphoma. Most of the existing literatures about primary pleural lymphoma are case reports. Most of them can be further divided into two subtypes: PEL and PAL (2). The main radiographic manifestation of PEL, as the name implies, is pleural effusion. Human herpes virus $8 /$ Kaposi sarcoma-associated herpes virus (HHV-8/KSHV) infection and immunosuppressive status such as HIV infection are commonly associated with the pathogenesis of PEL (4). PAL is triggered by long-standing empyema, which usually last for more than 20 years after the initial pneumothorax treatment for tuberculosis (5). Chronic inflammation and Epstein-Barr virus (EBV) infection are associated with PAL (6). Primary pleural lymphoma in immunocompetent patients without a history of pyothorax is extremely rare and there are few published reports (2). We report a case of primary pleural DLBCL without HIV infection. The patient had no history of tuberculosis, pyothorax, or pleural effusion. Inhomogeneous pleural thickening is the only radiographic manifestation. To the best of our knowledge, it's the first time to report this unique type of primary pleural lymphoma in China.

DLBCL is the most common type of aggressive nonHodgkin lymphoma, representing approximately 30-40\% of all cases (7). R-CHOP, a multi-drug rituximab-based chemoimmunotherapy regimen, is the standard therapy for DLBCL patients (8). The pathogenesis of primary pleural DLBCL is not yet elucidated and chronic inflammation is considered to be involved (9). According to the report of Iwasa et al. (1), we found only seven similar cases documented previously, which were non-pyothoraxassociated primary pleural DLBCL with pleural thickening or masses but without pleural effusion. All the cases were from Japan and the characteristics of them as well as our patient are summarized in Table 1. Of the eight reported cases, the average age is 60 years (17-84 years), and the ratios of males to females is $3: 5$. Their main radiographic finding is the pleural mass, with ribs involved in five cases. In our case, there was no bone involvement, while a vessel sign was visible in the lesion. The vessel was considered to be an intercostal artery that was enveloped by parietal pleural masses. That is called "sandwich sign" as described in two cases by Kim et al. and this characteristic CT sign would be helpful in differentiating primary pleural lymphoma from other pleural tumors (10).

There is little evidence available for the treatment of primary pleural DLBCL. Operation, chemotherapy, radiotherapy and combination of these were performed in previously reported cases. Of the eight cases, three received monotherapy of chemotherapy. The others received different combination therapies. Chemotherapy is frequently performed and usually indispensable for a combination therapy. Peyrade et al. (11) reported a median survival of 29 months and an actuarial two-year survival rate of $47 \%$ in DLBCL patients treated with R-CHOP. The most common chemotherapeutic side effect is myelosuppression. The prognosis is diverse. Two of the eight reported cases died within half a year and others were still alive with different survival time. We suppose that these differences might be attributed to different biochemical features of the malignancy, performance statuses (PS) of the patients, and their responses to treatments. We administered full-dose R-CHOP in our patient taking into account his good PS. He responded well to the chemotherapy except for mild myelosuppression, and he is still in follow-up. 
In conclusions, we report a very rare case of primary pleural DLBCL in an immunocompetent patient without history of empyema or pleural effusion. The pathogenesis of this disease is still unknown. The pleural thickening and mass, as well as occasional bone involvement are main CT findings of this unusual type of lymphoma. In clinical practice, pleural thickening is mainly seen in malignant mesothelioma and chronic pleural inflammation such as pleuritis. From our case and the review of the literature, the possibility of lymphoma cannot be neglected although it is a rare manifestation. Although a pleural "sandwich sign" may suggest this diagnosis, CT findings lack specificity in most cases. The differential diagnosis should include pleural metastasis and malignant mesothelioma. Percutaneous core needle biopsy or surgical biopsy can provide enough specimens for diagnosis. As an aggressive malignancy, early diagnosis and treatment are important for primary pleural DLBCL to improve patients' quality of life and prolong their survival.

\section{Acknowledgements}

We thank Dr. Dengbing Chen in the Department of Hematology-Oncology at our hospital for his contribution to the treatment strategy of the patient.

\section{Footnote}

Conflicts of Interest: The authors have no conflicts of interest to declare.

Informed Consent: Written informed consent was obtained from the patient for publication of this manuscript and any accompanying images.

\section{References}

1. Iwasa Y, Okada A, Takenaka H, et al. Primary Malignant

Cite this article as: Shao C, Guo Y, Xu X, Pan D, Wang B, Tang Y, Xu S. Non-pyothorax-associated primary pleural lymphoma without pleural effusion in an immunocompetent patient: a case report and literature review. J Thorac Dis 2018;10(5):E368-E371. doi: 10.21037/jtd.2018.04.163
Lymphoma Originating from the Chest Wall without Preceding Pleural Disease. Intern Med 2017;56:681-6.

2. Oikonomou A, Giatromanolaki A, Margaritis D, et al. Primary pleural lymphoma: plaque-like thickening of the pleura. Jpn J Radiol 2010;28:62-5.

3. Shah HJ, Keraliya AR, Jagannathan JP, et al. Diffuse Large B-Cell Lymphoma in the Era of Precision Oncology: How Imaging Is Helpful. Korean J Radiol 2017;18:54-70.

4. Okada S, Goto H, Yotsumoto M. Current status of treatment for primary effusion lymphoma. Intractable Rare Dis Res 2014;3:65-74.

5. Ueda T, Andreas C, Itami J, et al. Pyothorax-associated lymphoma: imaging findings. AJR Am J Roentgenol 2010;194:76-84.

6. Hibino $M$, Irie $T$, Ohe $M$, et al. Usefulness of diffusionweighted magnetic resonance imaging-guided biopsy: pyothorax-associated lymphoma. Intern Med 2015;54:2661-5.

7. Li S, Young KH, Medeiros LJ. Diffuse large B-cell lymphoma. Pathology 2018;50:74-87.

8. Chiappella A, Santambrogio E, Castellino A, et al. Integrating novel drugs to chemoimmunotherapy in diffuse large B-cell lymphoma. Expert Rev Hematol 2017;10:697-705.

9. Sun ML, Shang B, Gao JH, et al. Rare case of primary pleural lymphoma presenting with pleural effusion. Thorac Cancer 2016;7:145-50.

10. Kim Y, Lee M, Ryu YJ, et al. The pleural sandwich sign in two cases of primary pleural lymphoma. Korean J Radiol 2015;16:213-6.

11. Peyrade F, Jardin F, Thieblemont C, et al. Attenuated immunochemotherapy regimen (R-miniCHOP) in elderly patients older than 80 years with diffuse large B-cell lymphoma: Amulticentre, single-arm, phase 2 trial. Lancet Oncol 2011;12:460-8. 